\title{
Publisher Correction: Generalized parity-time symmetry condition for enhanced sensor telemetry
}

Pai-Yen Chen, Maryam Sakhdari, Mehdi Hajizadegan, Qingsong Cui, Mark Ming-Cheng Cheng, Ramy El-Ganainy and Andrea Alù iD

Correction to: Nature Electronics https://doi.org/10.1038/s41928-018-0072-6, published online 14 May 2018.

In the version of this Article originally published, a division symbol was mistakenly omitted from both of the $y$ axis labels in Fig. 5a. The label in the left panel should have read ' $\operatorname{Re}\left(\left(\omega \times \omega_{0}\right) / 2 \pi\right)(\mathrm{MHz})^{\prime}$ and the label in the right panel should have read ' $\operatorname{Im}\left(\left(\omega \times \omega_{0}\right) / 2 \pi\right)(\mathrm{MHz})$ '.

This has now been corrected. 